\title{
Experimental Study of Utility Function Selection for Video over IEEE 802.22 Wireless Regional Area Networks
}

\author{
${ }^{\dagger}$ Youping Zhao, ${ }^{\ddagger}$ Shiwen Mao, ${ }^{J}$ Jeffrey H. Reed, and ${ }^{\ddagger}$ Yingsong Huang \\ ${ }^{\dagger}$ Shared Spectrum Company, Vienna, VA 22182 \\ ${ }^{\ddagger}$ Dept. Electrical and Computer Engineering, Auburn University, Auburn, AL 36839-5201 \\ $\S$ The Bradley Dept. Electrical and Computer Engineering, Virginia Tech, Blacksburg, VA 24061 \\ Email: yzhao@sharedspectrum.com, smao@ieee.org, reedjh@vt.edu, yzh0002@auburn.edu
}

\begin{abstract}
Cognitive Radio (CR) is a new wireless communications and networking paradigm that is enabled by the Software Defined Radio (SDR) technology and a recent change in spectrum regulation policy. As the first commercial application of CR technology, IEEE 802.22 wireless regional area networks (WRAN) aim to offer broadband wireless access by efficiently utilizing "white spaces" in the broadcast TV bands. In this paper, we evaluate the performance of an IEEE 802.22 WRAN base station (BS) cognitive engine (CE) testbed developed at Wireless@Virginia Tech on supporting video applications. We investigate the important problem of utility function selection and its impact on the received video quality. Through testbed experiments, we find that a video-specific utility function achieves significant improvements on received video quality over a general purpose utility function, indicating the efficacy of cross-layer design and more importantly, the need for adopting dynamic situation- and application-aware utility functions at the $C E$, rather than a predefined static one.
\end{abstract}

\section{INTRODUCTION}

Cognitive Radio (CR) is a new wireless communications and networking paradigm that is enabled by the Software Defined Radio (SDR) technology [1] and a recent change in spectrum regulation policy [2]. A CR node can sense the spectrum to detect unused frequency bands (white space). Its frequency-agile radio module can be tuned to a detected unused band and operate from there. The CR concept represents a significant paradigm change from exclusive use of spectrum by licensed users (or, primary users), to dynamic spectrum access for unlicensed users (or, secondary users). It may change the way how wireless communications systems are designed, and drive the next generation of radio devices and wireless standards to enable a variety of new applications.

The high potential of CR has attracted significant research efforts [3], [4]. A particular progress was made with the formation of the IEEE 802.22 working group in 2004, aiming to develop standards for point-to-multipoint wireless regional area networks (WRAN) for utilizing UHF/VHF TV bands ranging from $54 \mathrm{MHz}$ to $862 \mathrm{MHz}$ for communications. The IEEE 802.22 WRANs are motivated by the fact that most of the TV programs are now provided via cable or satellite networks, and the broadcast TV bands are usually underutilized. In addition, the use of TV channels by primary users are highly predictable (i.e., regular program hours and fixed number of channels for a long period of time), making it relatively easier for secondary users to detect the presence of primary users and to access the spectrum. A typical IEEE 802.22 system consists of one or more base stations (BS) and customer premises equipments (CPE). The CPEs will sense the channel and report the sensing results to the BS periodically. The BS, based on collected channel information, will intelligently allocate radio resources to the CPEs to satisfy their quality of service (QoS) requirements, under constraints such as the maximum transmit power and emission masks [5].

Although SDR makes waveforms programmable, it is the intelligence, awareness, and learning capability of the CR that fully exploits its potential. The "intelligent agent" that manages cognition tasks in a CR is called a cognitive engine (CE) [6]. By leveraging past experience and knowledge, the $\mathrm{CE}$ can choose the most efficient reasoning and learning method and make (near-)optimal and/or cross-layer adaptations subject to constraints of regulation, policy, and radio equipment capability. The CE usually takes into account the most pertinent performance metric(s) and incorporates them into a proper utility function to meet the CR's goal for the specific radio scenario or application. Therefore, the choice of performance metrics and utility functions is critical for CR performance.

In this paper, we evaluate the performance of an IEEE 802.22 WRAN BS CE testbed developed at Wireless@Virginia Tech on supporting video applications [7]-[9]. Video is one of the killer applications for numerous emerging (cognitive) wireless networks. As a bandwidth-hungry and distortion sensitive application, radio resource management needs to be highly efficient and intelligent among network nodes in light of the demanding (perhaps also dynamically changing) system requirements and constraints. We assume an IEEE 802.22 WRAN with a BS and multiple CPEs, while the BS is transmitting a video stream to each CPE. We assume certain feedback and rate control mechanism between the BS and the corresponding video servers, such that each server can change the bit rate of the streaming video based on feedback from the BS, in order to fully exploit the available bandwidth in 
the WRAN and to maximize the received video quality at the CPEs.

Specifically, we investigate the important problem of utility function selection and its impact on the received video quality. Utility functions play an important role in the convergence speed and final achievable performance of cognitive wireless networks [6]. Through testbed experiments, we compare the performance, in the form of received video distortion, achieved when two different utility functions are used in the CE. The first utility function is a generic utility function defined for supporting a variety of services to subscribers such as voice, data, and video. The second utility function is in the form of endto-end video distortion consisting of the encoder distortion caused by quantization and channel distortion due to packet loss within the network [10]-[13]. We find that the videospecific utility function achieves significant improvements on received video quality, indicating the efficacy of cross-layer design and more importantly, the need for adopting dynamic situation- and application-aware utility functions at the CE, rather than a predefined static one.

The remainder of this paper is organized as follows. We review the WRAN BS CE testbed architecture and key system parameters in Section II. We then define the utility functions employed in the testbed experiments in Section III. Our experimental results are present in Section IV. Section V discusses related work and Section VI concludes this paper.

\section{WRAN BS CE TeStbed ARCHITECTURE AND KeY PARAMETERS}

As discussed, IEEE 802.22 WRAN is the first commercial application of CR technology, aiming to offer broadband wireless access by efficiently utilizing "white spaces" in the broadcast TV bands [5], [14]. In this section, we describe the architecture and key system parameters of a WRAN BS CE testbed developed at Wireless@ Virginia Tech [7]-[9], which will be used in our experimental study of video over CR networks.

As shown in Fig. 1, the WRAN BS consists of a spectrum sensing module, a Radio Environment Map (REM), the CE, and a software defined radio (SDR) transceiver. The spectrum sensing module is used to sense the presence of primary users in the neighborhood, while the sensing results will be recorded in the REM. The REM is basically a comprehensive database designed for providing network-wide situation-awareness [15], [16]. The CE, as the "brain" of network radio resource management, may incorporate various learning and reasoning methods such as case- and knowledge-based learning (CKL), a channel modeler and predictor, a multi-objective optimizer, a spectrum manager and other functional entities [7]-[9]. The CE first obtains situation awareness by spectrum sensing (i.e., any active primary user in the neighborhood?) and by querying the REM. It then determines the utility function that best fits the current situation for radio resource management [6], [9].

The multi-objective optimizer in the $\mathrm{CE}$ is implemented with genetic algorithms (GA) [17]. GA is a population-based metaheuristic inspired by the survival-of-the-fittest principle.

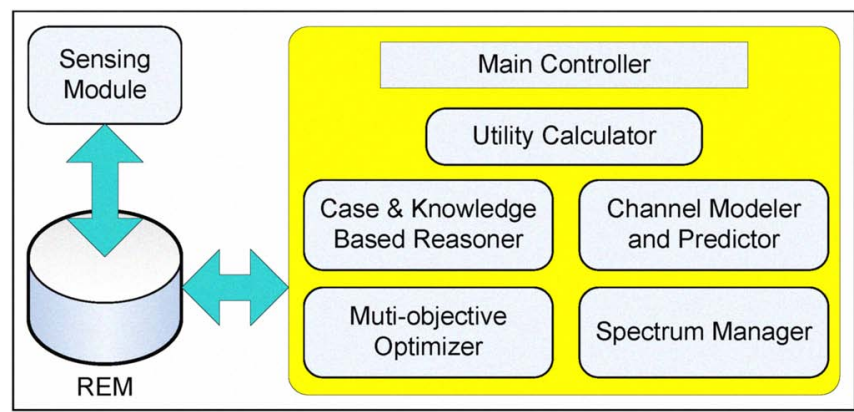

Fig. 1. Architecture of the REM-based CE for an IEEE 802.22 WRAN BS.

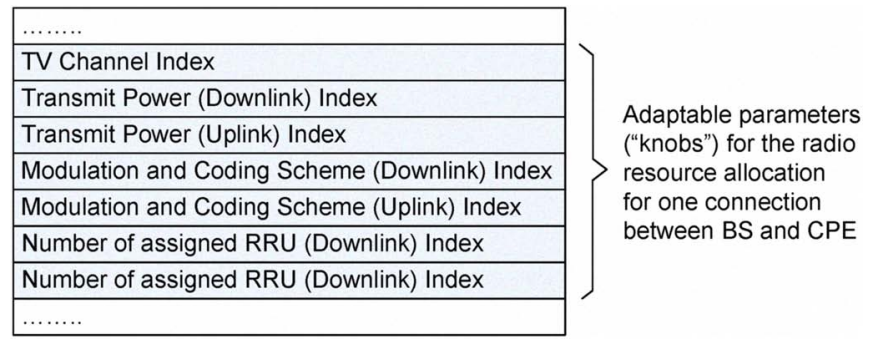

Fig. 2. A section of the chromosome for the GA-based WRAN CE.

GA is particularly suitable for solving complex combinatorial optimization problems, most of which are multi-modal and non-convex. In GA, a solution is coded in the form of chromosome, and several genetic operators such as crossover and mutation are adopted to change the chromosomes to guide the search in the solution space, while selection of competitive solutions is based on their fitness (in the form of an objective, or utility function). The survival-of-the-fittest principle ensures that the overall quality of the population improves as the algorithm progresses from one generation to the next.

A section of the chromosome for the GA-based WRAN $\mathrm{CE}$ is shown in Fig. 2. The size of the chromosome (in bits), and hence the computational complexity, increases with the number of new connections, the number of adaptable parameters ("knobs") of the CR node, and the adaptation range of each "knobs." The GA-based multi-objective optimizer helps the CE to achieve multiple goals (such as minimizing harmful interference and maximizing spectrum efficiency) subject to various practical constraints and regulations (such as the maximum transmit power and emission masks) [5].

For real-world radio scenarios of WRAN systems, the distributed CPEs may have various locations and different types of service requests (such as voice, data, and video). To facilitate efficient radio resource management, the WRAN BS CE needs to know the amount of radio resource that is required to setup a new connection for a CPE. To obtain a generic and convenient measure of the available radio resource at the BS and the requested radio resource from CPEs, a unitless metricRadio Resource Unit (RRU)-is employed in the WRAN BS $\mathrm{CE}$ testbed. For example, the required RRU $\left(R R U_{r e q}\right)$ for a 
TABLE I

SPECTRAL EFFICIENCY OF SEVERAL Modulation AND CODING SCHEMES FOR WRAN

\begin{tabular}{|l|c|c|}
\hline Modulation Level & Coding Rate & $\begin{array}{c}\text { Spectral Efficiency } \\
\text { (bps/Hz) }\end{array}$ \\
\hline \hline QPSK & $1 / 2$ & 1 \\
\hline QPSK & $3 / 4$ & 1.5 \\
\hline QPSK & 1 (no coding) & 2 \\
\hline 16QAM & $1 / 2$ & 2 \\
\hline 16QAM & $3 / 4$ & 3 \\
\hline 16QAM & 1 (no coding) & 4 \\
\hline 64QAM & $2 / 3$ & 4 \\
\hline 64QAM & $3 / 4$ & 4.5 \\
\hline 64QAM & 1 (no coding) & 6 \\
\hline
\end{tabular}

new connection can be estimated by

$$
R R U_{r e q}=(1+\alpha) \frac{R}{\eta \cdot B W_{s c}},
$$

where $\alpha$ is the overhead factor (unitless) that accounts for the overhead of the WRAN protocol and can be determined from the WRAN system specification; $R$ is the data rate of the new connection (unit: bps) and determined by the service type; $\eta$ is the spectral efficiency (unit: $\mathrm{bps} / \mathrm{Hz}$ ) jointly determined by the highest applicable modulation level and channel coding rate; $B W_{s c}$ is the bandwidth of the WRAN OFDM sub-carrier (unit: $\mathrm{Hz}$ ) defined as

$$
B W_{s c}=\frac{\text { TV Channel Bandwdith }}{\text { FFT Mode }} .
$$

More specifically, in the context of the OFDM modulation format assumed for the WRAN, the physical meaning of $R R U_{r e q}$ is the number of OFDM sub-carriers to be allocated per WRAN frame for a given service request from the CPE. Eqn. (1) takes into account the WRAN protocol overhead. Intuitively, the larger the associated protocol overhead, the more RRUs will be required. The spectral efficiency of several typical modulation and coding schemes used in WRAN systems are given in Table I.

Considering the inherent cognition and interference avoidance capability of WRANs, the current version of the WRAN BS CE testbed estimates the SNR without considering any type of interference. The modulation and coding scheme is selected based on a rough SNR estimation at the receiver under additive white Gaussian noise (AWGN) channels assuming the radio link between the BS and CPE is (quasi-) static.

\section{Definition OF Utility FunCtions}

As discussed, utility functions are used in the CE for radio resource management. The choice of utility functions is critical for the CE to achieve the desired CR performance, since they determine the convergence and optimality of the solutions produced by the multi-objective optimizer in the CE. In this section, we describe two types of utility functions for the WRAN BS CE testbed, a generic utility function that can be used for general WRAN applications and an applicationspecific utility function for video over CR networks.

\section{A. Performance Metrics and the Global Utility Function}

The first step is to choose or define proper performance metrics for $\mathrm{CE}$, which can then be incorporated into a utility function. The following performance metrics are adopted for the WRAN BS CE testbed [6]-[8].

- $u_{1}=$ QoS satisfaction of all connections, in terms of the average utility of all downlink and uplink connections between CPEs and the BS.

- $u_{2}=$ spectral efficiency, in terms of the number of candidate channels or the total amount of RRU assigned per active TV channel. This metric is more important for multi-cell scenarios or a single cell with a large number of CPEs.

- $u_{3}=$ power efficiency, in terms of the transmit power of individual CPEs. This metric is more important for mobile or portable user devices or overlapping WRANs operated by different service providers.

The utility function employed by the CE is usually defined as a weighted combination of multiple selected performance metrics. To be flexible for various radio scenarios or applications, a viable approach for the $\mathrm{CE}$ is to adopt dynamic situation-aware utility functions rather than a predefined static one. In general, the global utility function for the WRAN BS $\mathrm{CE}$ can be defined as

$$
u_{\text {global }}=\prod_{i}\left(u_{i}\right)^{\omega_{i}},
$$

where $\omega_{i}$ is the weight applied to the $i$-th performance metric $\left(u_{i}\right)$. Different weight vectors could be applied to adjust the utility function. Similar to the geometric mean, this definition of $u_{\text {global }}$ accentuates low utility metrics, thus providing a fair and balanced combination of various performance metrics.

For the WRAN BS CE testbed, the global utility $\left(u_{\text {global }}\right)$ is subdivided between individual CPE utilities $\left(u_{c p e}\right)$ and the normalized spectral efficiency of the BS $\left(u_{B S}\right)$ as

$$
u_{\text {global }}=\left[\prod_{i}^{N}\left(u_{c p e, i}\right)^{\omega_{c p e} / N}\right] \cdot\left(u_{B S}\right)^{\omega_{B S}},
$$

where $N$ is the number of active CPEs currently associated with the WRAN BS, and $\omega_{c p e}$ and $\omega_{B S}$ are the weight for the geometric mean of individual CPE utilities and the weight for the spectral efficiency of the BS, respectively. The weights $\left(\omega_{c p e}\right.$ and $\left.\omega_{B S}\right)$ can be determined by the WRAN operator based on its priority and goal. For the experiments reported in this paper, $\omega_{\text {cpe }}$ is set to 0.9 and $\omega_{B S}$ is set to 0.1 . In the testbed, the global utility function (3) is employed by the GA-based CE to evaluate the overall "fitness" of a set of radio resource configuration parameters as listed in Fig. 2.

\section{B. A Generic Utility Function for CPEs}

To accommodate multiple applications, a generic utility function proposed for individual CPE is defined as

$$
\begin{aligned}
& u_{c p e}= \\
& {\left[f_{1}\left(P_{b}^{-1}, P_{b_{0}}^{-1}\right)\right]^{0.5}\left[f_{2}\left(R_{b}, R_{0}\right)\right]^{0.4}\left[f_{3}\left(P_{t}^{-1}, P_{t_{0}}^{-1}\right)\right]^{0.1}}
\end{aligned}
$$




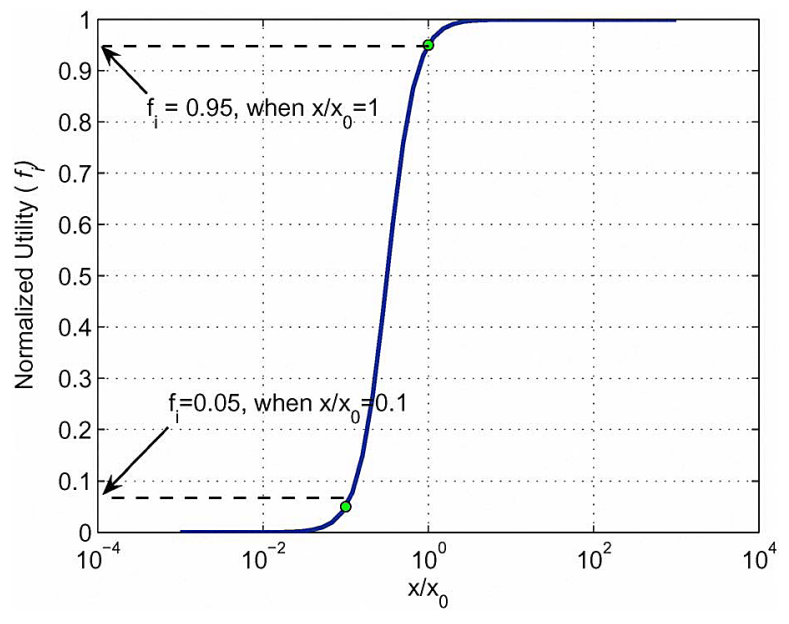

Fig. 3. Illustration of the CPE utility function $f_{i}$, a modified hyperbolic tangent function.

where $P_{b}, R_{b}$, and $P_{t}$ are the measured or estimated bit-errorrate, data rate, and transmit power of the CPE, respectively; and $P_{b_{0}}, R_{0}$, and $P_{t_{0}}$ are the target bit-error-rate, data rate, and transmit power of the CPE, respectively. The utility function $f_{i}$ 's are modified (shifted and spread/compressed) hyperbolic tangent functions, i.e.,

$$
\begin{aligned}
& f_{i}\left(x, x_{0} ; \eta_{i}, \sigma_{i}\right)= \\
& \frac{1}{2}\left\{\tanh \left[\log \left(\frac{x}{x_{0}}\right)-\eta_{i}\right] \cdot \sigma_{i}+1\right\}, i=1,2,3,
\end{aligned}
$$

where $x$ and $x_{0}$ are the performance metric and the target value, respectively; and $\eta_{i}$ and $\sigma_{i}$ are the threshold and the spread parameter, respectively. The utility function $f_{i}$ is monotone and bounded by 0 and 1 , as shown in Fig. 3. For the WRAN BS CE testbed, the threshold $\left(\eta_{i}\right)$ and spread parameter $\left(\sigma_{i}\right)$ are chosen such that

- the utility is 0.95 when the metric $(x)$ achieves the target value $\left(x_{0}\right)$, and

- the utility is 0.05 when the metric is one decade below the target value.

Note that the individual CPE utility function (5) represents the degree of satisfaction of the user to the overall radio resource management. The modified hyperbolic tangent function is a type of sigmoid function that can accommodate a large range of performance variations and capture the value of the service to the user quite naturally. If the solution does not meet the target goal, the utility is decreased sharply. On the other hand, since solutions that result in excessively high QoS provide little extra value to the user, the increase of utility is marginal when $x$ is within this range.

\section{A Video-Specific Utility Function for CPEs}

Our second choice of utility function for CPEs is a video application-specific one, which is defined as a function of the estimated mean squared error (MSE) distortion based on an empirical rate-distortion model for streaming videos. We

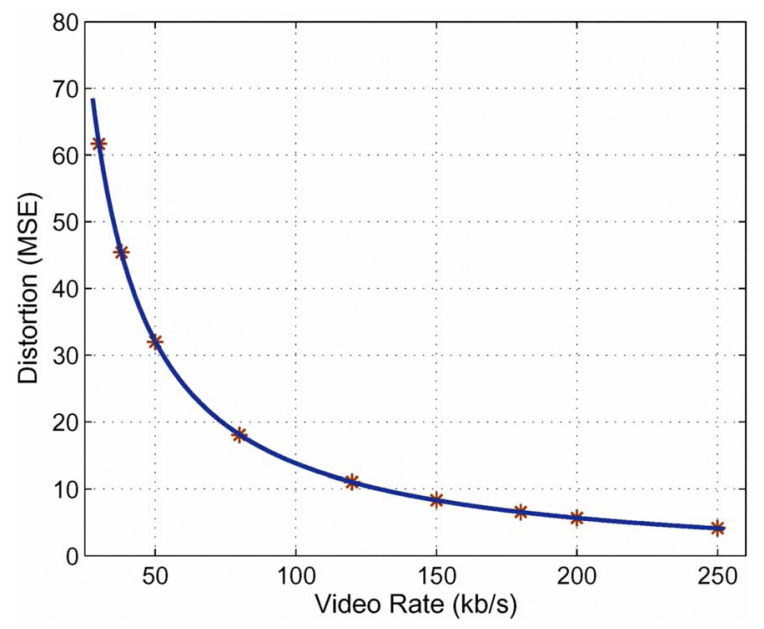

Fig. 4. Rate distortion curve at the encoder for the QCIF test sequence Foreman, which is encoded with an H.264 codec at 15 frames per second. The markers are measured points, while the curve is computed using (7).

consider the distortion of a received video, which largely consists of the encoder distortion $\left(D_{e}\right)$, caused by quantization, and channel distortion $\left(D_{c}\right)$ due to packet loss within the network [10], [11]. The end-to-end video distortion is

$$
D=D_{e}+D_{c} \text {. }
$$

Based on the rate-distortion theory and experimental studies, many empirical models have been introduced in the literature [10], [11], [18]-[21]. We adopt the model proposed in [10] in this paper without loss of generality. Under this model, the encoder distortion, measured as MSE, can be evaluated by:

$$
D_{e}=\phi+\frac{\theta}{R-\lambda},
$$

where $\phi, \theta$ and $\lambda$ are constants for a specific video codec and video sequence, and $R$ is the coding bit rate. We verify this model using the H.264 codec with options in the Baseline profile and the Quarter Common Intermediate Format (QCIF) Foreman test sequence. The measured distortion and the computed distortion using (7) are plotted in Fig. 4, where excellent match can be observed.

The second component of $D$, channel distortion $D_{c}$ is caused by packet loss within the network. We adopt the general model presented in [11] for channel distortion, which has the following simplified form (i.e., an upper bound):

$$
D_{c}=\frac{p}{\beta \cdot(1-p)} D_{E C P},
$$

where $D_{E C P}$ is a constant and can be predetermined by training; $\beta$ is the intra rate, a coder parameter; and $p$ is the average packet error rate (PER).

The parameters for the QCIF Foreman video sequence used in our experiments are given in Table II. We then adopt a video application-specific utility function for the CPEs, which is defined as follows:

$$
u_{\text {cpe }}= \begin{cases}1 / D, & \text { if } D>1 \\ 1, & \text { if } D \leq 1 .\end{cases}
$$


TABLE II

PARAMETERS FOR THE Foreman VIDEO SEQUence UsEd IN OUR EXPERIMENTS

\begin{tabular}{|l||c|c|c|c|c|}
\hline Parameter & $\phi$ & $\theta$ & $\lambda$ & $D_{E C P}$ & $\beta$ \\
\hline Value & -1.783 & 1411 & 7.046 & 50 & 0.03 \\
\hline
\end{tabular}

Maximizing this utility for the CPEs is equivalent to minimizing the distortion of received videos at the CPEs.

\section{The BS Utility Function}

The normalized BS spectral efficiency $\left(u_{B S}\right)$ is determined by averaging the number of available subcarriers per WRAN channel, i.e.,

$$
u_{B S}=\frac{1}{M} \sum_{i=1}^{M} u_{B S}^{i}
$$

where $M$ is the number of channels supported by the BS, and $u_{B S}^{i}$ is the spectral efficiency of the $i$-th WRAN channel, which also indicates the radio resource utilization of this channel at the BS, $i=1,2, \cdots, M$.

For the current version of the WRAN BS CE, $u_{B S}^{i}$ is defined as

$$
u_{B S}^{i}=1+\tanh \left(\frac{R R U_{\text {available }}-R R U_{\text {capacity }}}{\sigma_{R R U}}\right),
$$

where $R R U_{\text {available }}$ is the number of RRUs that are available for the $i$-th WRAN channel at the BS, ranging from 0 to $R R U_{\text {capacity }} ; R R U_{\text {capacity }}$ is the maximal number of subcarriers available for a WRAN channel; and $\sigma_{R R U}$ is the spread parameter for the modified hyperbolic tangent function.

In the WRAN BS CE testbed, $R R U_{\text {capacity }}$ for a WRAN channel is set to 2048, while $\sigma_{R R U}$ is set to 800 . It can be verified that $u_{B S}^{i}$ is monotone and is bounded by 0 and 1 . The rationale to adopt such a modified hyperbolic tangent function as the utility function for overall WRAN BS spectrum efficiency, is that it helps the CE to squeeze the spectrum used by the WRAN BS (in terms of the number of channels or subcarriers in use) through the optimization process. For example, the solution, which assigns the CPEs to subcarriers spread into two or more WRAN channels, will produce a lower BS utility $\left(u_{B S}\right)$ as compared to the more spectral efficient solution in which the CPEs are assigned to subcarriers within the same WRAN channel.

\section{EXPERIMENTAL SySTEM PARAMETERS AND RESUlTS}

\section{A. Testbed Setting}

To evaluate the performance of the WRAN CE with different utility functions, we adopt a typical WRAN scenario where forty (40) CPEs are randomly distributed in the service area of one WRAN BS. As shown in Fig. 5, the WRAN BS is located at the origin, while the $40 \mathrm{CPEs}$ are randomly distributed in the $33 \mathrm{~km} \times 33 \mathrm{~km}$ network region. The simulation parameters for the WRAN BS CE testbed are presented in Table III, and the requested service at each CPE is set to "video." The adjustable parameters of the testbed are listed in Table IV.

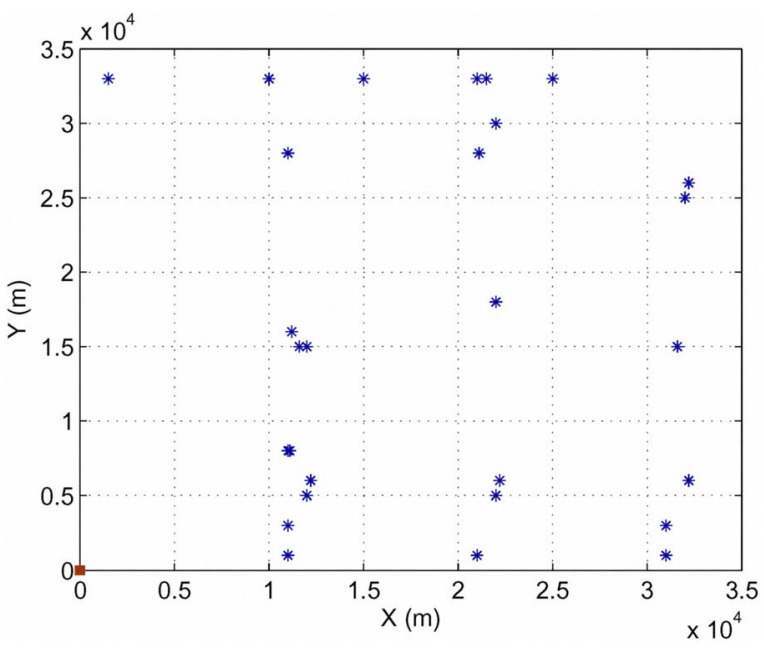

Fig. 5. The WRAN network used in our experiments. The stars are CPEs randomly located in the $33 \mathrm{~km} \times 33 \mathrm{~km}$ area, while the WRAN BS is located at the origin.

For the results reported in this section, an H.264 reference software encoder in the Baseline profile is used to encode the test video sequence in realtime. Each encoded video frame is carried in one UDP packet, so that a lost packet leads to a lost frame [11]. Without loss of generality, we use the medium motion test sequence Foreman in the QCIF format. The videos are encoded at 15 frames/s, while the first frame is coded in the intra-mode (i.e., an I frame) and all the remaining frames are $\mathrm{P}$ frames. The intra rate is $\beta=0.03$. The distortion of the received videos are determined by the average channel distortion due to packet loss and encoder distortion, both in MSE, as given in (6).

\section{B. Experiment Results}

Two sets of experiments are carried out to evaluate the impact of the two CPE utility functions under the same network condition. In each experiment, the $\mathrm{CE}$ is used to allocate radio resource to the CPEs for streaming H.264 encoded videos to each of them. During video streaming, we record the utility, average distortion of received video, average video bit rate, average video packet loss rate, and the allocated transmit power for each CPE at the WRAN BS. The experiment results are shown in Figs. 6-10.

In Fig. 6, we plot the CPE utilities achieved by both CPE utility functions. Specifically, Fig. 6(a) is for the case when two WRAN channels are available, while Fig. 6(b) is for the case when three WRAN channels are available. In both cases, we find that the video utility of each CPE is fairly low (less than 0.1 for most CPEs) when the generic utility function as defined in (4) is used, while using the video-specific utility function as defined in (9) can significantly increase the video utility at each CPE. For most CPEs, there is over 10 times improvement in video utility achieved by using the video-specific utility function. It is also interesting to note that using more WRAN channels does not significantly improve the video utility at 
TABLE III

EXPERIMENTAL PARAMETERS FOR THE WRAN BS CE TESTBED

\begin{tabular}{|l|l|}
\hline Parameter & Value or Range \\
\hline \hline Cell radius & $33 \mathrm{Km}$ \\
\hline Types of service and quality of service requested by the CPEs & - Voice: target rate $-10 \mathrm{kbps}$, target BER $-10^{-2}$ \\
& - VIdeo: target rate $-100 \mathrm{kbps}$, target BER $-10^{-3}$ \\
& - Low data rate: target rate $-250 \mathrm{kbps}$, target BER $-10^{-6}$ \\
& - High data rate: target rate $-750 \mathrm{kbps}$, target BER $-10^{-6}$ \\
\hline Multiplexing/duplexing & OFDMA/TDD \\
\hline Number of subcarriers per channel (FFT mode) & 2,048 \\
\hline WRAN channel bandwidth & $6 \mathrm{MHz}$ \\
\hline
\end{tabular}

TABLE IV

ADJUSTABLE PARAMETERS (“KNOBS") AT THE WRAN BS AND CPES

\begin{tabular}{|l|l|}
\hline Parameter & Value or Range \\
\hline \hline Channel frequency & VHF/UHF (54 - 862 MHz) \\
\hline Transmission power of BS, CPE & $\begin{array}{l}\text { Up to 4 Watts, subject to the RF emission mask defined by the } \\
\text { IEEE 802.22 standards and local regulators }\end{array}$ \\
\hline Modulation Schemes & QPSK, 16QAM, and 64QAM \\
\hline Channel coding & None, 1/2, 2/3, and 3/4 (convolutional coding rate) \\
\hline Number of UL/DL subcarriers allocated to the new connection & Variable from 4 to 256 \\
\hline
\end{tabular}

the CPEs under the generic utility function, while the videospecific utility function can better exploit the increased radio resource to achieve higher utility for most of the CPEs. It can be seen with three WRAN channels, most of the CPEs achieves the maximum utility of 1 when the video-specific utility function is used.

We next plot the average distortion (MSE) of the received videos at the CPEs in Fig. 7, where two WRAN channels are used. As expected, we find the video distortion at each CPE is significantly reduced when using the video application-specific utility function, as compared to that when using the original generic utility function. In the first case, video distortion is less than 10 for most CPEs, implying excellent perceived video quality at the CPEs. Similar observation can be made in the case of three WRAN channels, but are omitted for brevity.

As shown in (6), the distortion of decoded video is mainly a function of the video bit rate $R$ and the PER $p$. To further illustrate the different performance of the two utility functions, we plot the average PER in Fig. 8 and the average video bit rate in Fig. 9 for each CPE. The PER is estimated at the CE as

$$
P E R=1-(1-B E R)^{L},
$$

where $B E R$ is the average bit error rate estimated at the CE according to the channel model and modulation and coding schemes, and $L$ is the average packet length in bits. Note that we assume that bit errors are uniformly distributed under the AWGN channel. We find in Fig. 8 that the PER at each CPE can be significantly reduced (less than $10^{-3}$ for most CPEs) when using the video-specific utility function, as compared to the case of using the generic utility function. Similarly, we find in Fig. 9 that the assigned bit rate for each CPE can be much higher (more than $200 \mathrm{kbps}$ for most CPEs) when using the video-specific utility function, as compared to the case of using the generic utility function. The joint effect of the increased bit rate and reduced PER is the improved video quality at each CPE, as observed in Fig. 7.

Finally, in Fig. 10 we plot the transmit power allocation for each CPE at the BS for the case of two WRAN channels and the case of three WRAN channels. It can be seen that the transmit power is higher for most of the CPEs when the videospecific utility function is used. This indicates that, however, the improvement in video utility achieved by the video-specific utility function is at the cost of higher transmission power at the BS. Such power consumption penalty is even more pronounced when fewer WRAN channels are available. This is due to the fact that transmit power is included for optimization under the generic utility function, but not considered in the video-specific utility function. However, for some radio device (such as BS and CPE in IEEE 802.22 WRAN systems, which usually have AC power supply), the increased power consumption might not be a limiting factor. For battery-powered mobile subscribers, a trade-off comes into play among transmission power, video bit rate (bandwidth), and video distortion.

The above comparisons under two WRAN channels are summarized in Table V. To further demonstrate the visual effect, we plot Frame 68 of the decoded video, when the two utility functions are used. Specifically, we show the worst, average, and best cases among all the CPEs for the experiments with each utility function. The visual difference in the quality of the decoded frames is obvious. In addition, the difference between Fig. 11(d) and Fig. 11(f) are quite small. This indicates that all the CPEs receive comparable video quality when the video-specific utility function is used, i.e., better fairness among the CPEs.

\section{RELATED WORK}

The concept of utility was initially introduced in economics, which is a generalized term for the satisfaction obtained by an individual from the "use" of a product or a service measured 
TABLE V

PERFORMANCE COMPARISON WHEN USING DIFFERENT UTILITY FUNCTIONS

\begin{tabular}{|l|l|l|}
\hline & The video-specific utility function (9) & The generic utility function (4) \\
\hline \hline Average data rate & $389 \mathrm{kbps}$ & $187 \mathrm{kbps}$ \\
\hline Average PER & $1.1234 \times 10^{-4}$ & 0.09 \\
\hline Average Video Distortion & 3.68 & 143.63 \\
\hline Average CPE Video Utility & 0.59 & 0.11 \\
\hline Average transmit power & $1.875 \mathrm{dBW}$ & $-1.95 \mathrm{dBW}$ \\
\hline
\end{tabular}

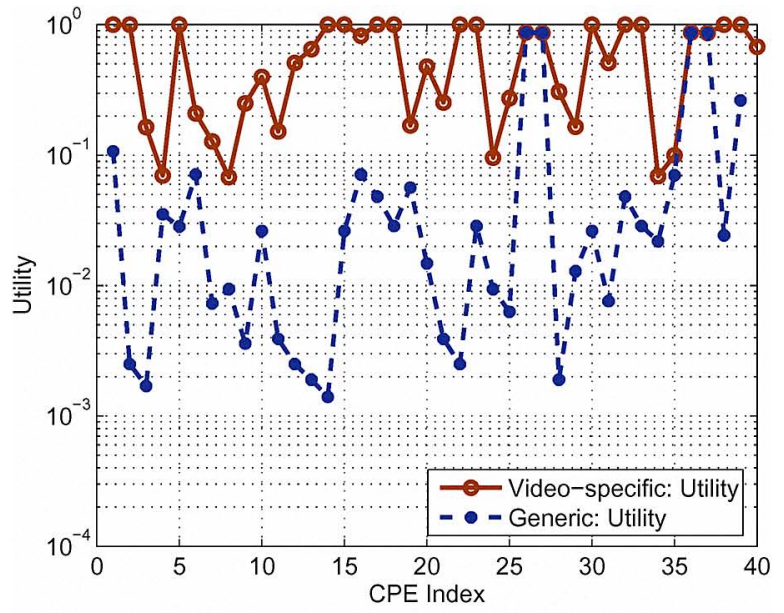

(a) Two WRAN channels

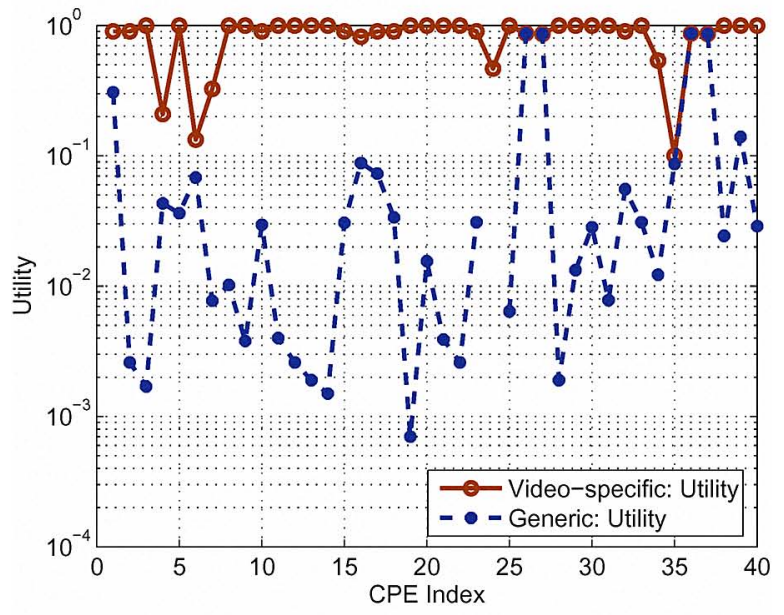

(b) Three WRAN channels

Fig. 6. Utilities at each CPE when using the generic utility function defined in (4) and when using the video-specific utility function defined in (9).

by the price that the individual is willing to pay [22]. In context of CR networks, utility is an assignment of values (numbers) to the current operating state such that the closer the cognitive radio comes to satisfying some goal, the greater the value assigned to the operating state [6]. Utility functions can incorporate a number of performance metrics and are usually dynamic and application-specific. Defining proper utility functions for wireless video networks is a very challenging and

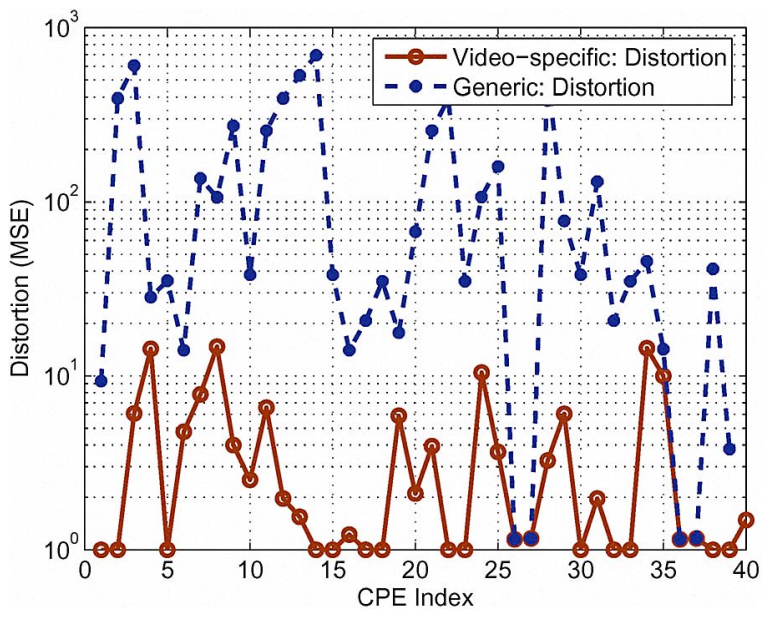

Fig. 7. Comparison of video distortions achieved by the two utility functions.

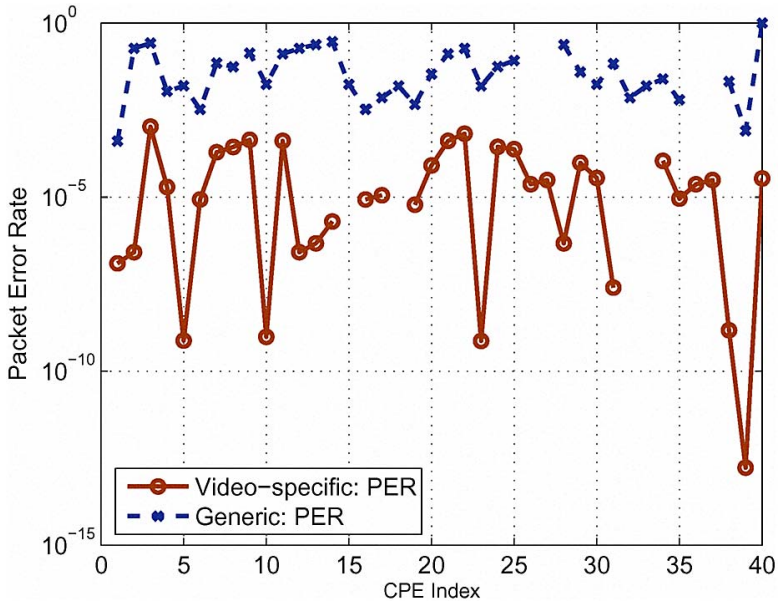

Fig. 8. Comparison of packet error rates (PER) achieved by the two utility functions.

complicated issue, evidenced by the fact that disparate ways have been used to measure the utility of streaming video (for more details, see [22] and the references therein).

Partially due to the lack of creditable simulation tools for cognitive radio networks, testbed is usually developed to evaluate/validate various signal processing techniques and CE algorithms or protocols in CR networks. Quite a few CR testbeds of various levels of cognition capability have been developed and reported in recent years [23]-[27]. In addition, 


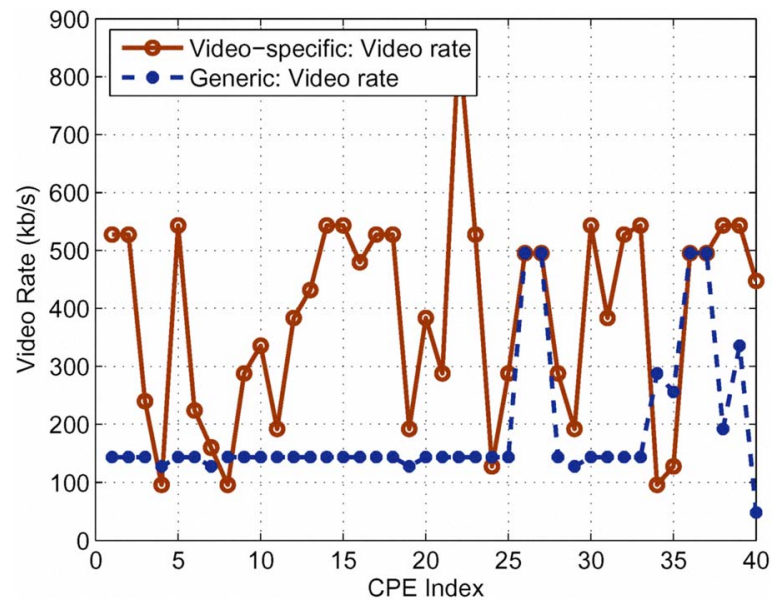

Fig. 9. Comparison of video bit rates achieved by the two utility functions.

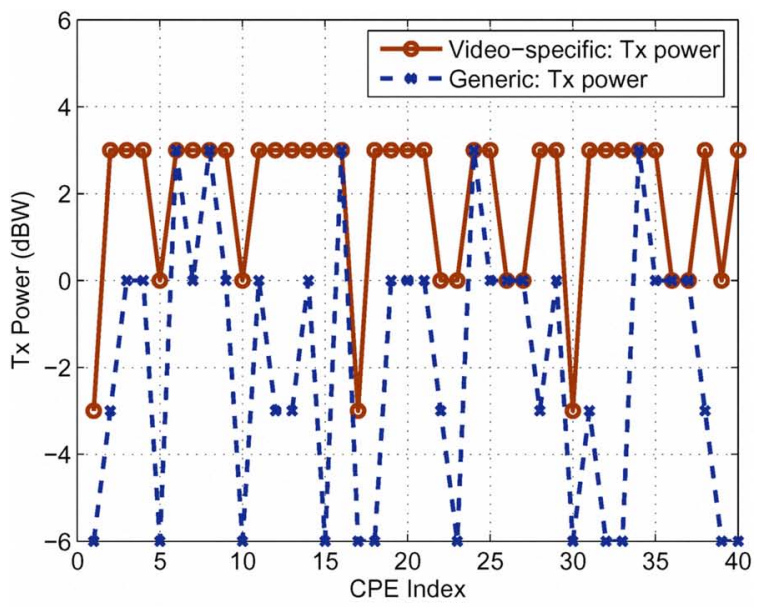

(a) Two WRAN channels

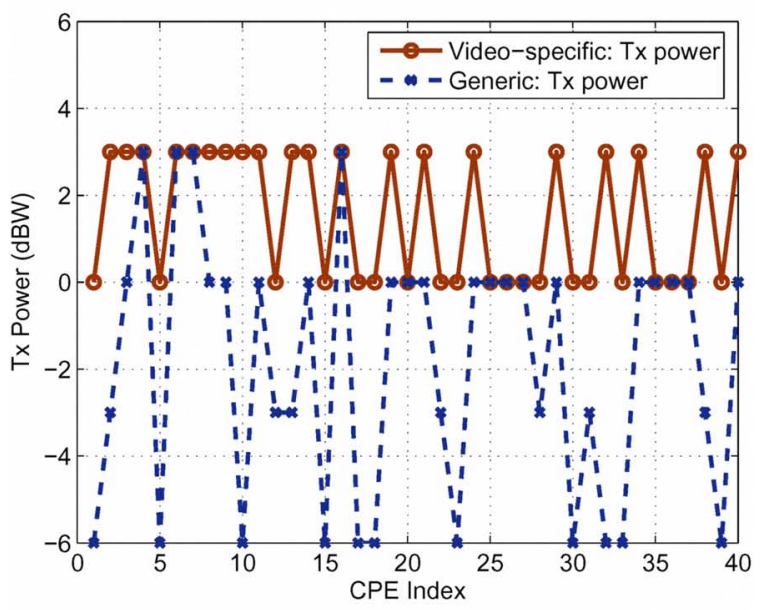

(b) Three WRAN channels

Fig. 10. Comparison of transmit powers when the two utility functions are used for CPEs. several CR testbeds (prototype or early-stage product) have been demonstrated at international conferences such as IEEE DySPAN 2008 and the 2008 Software Defined Radio Forum Technical Conference and Product Exposition, featuring dynamic spectrum access, cognitive radio device utilizing TV white space, network interoperability, and policy-based systems.

Video over wireless networks has become a very active research area attracting considerable efforts from the research community. Cross-layer optimization is an effective and important technique for providing satisfactory video quality in both infrastructure-based wireless networks [28], [29] and multi-hop wireless networks [12], [13], [21], [30], where the received video distortion is minimized by jointly considering parameters/mechanisms in multiple layers. The main difference between this paper and the existing work is that unlike in the prior work where the spectrum is exclusively used by the video sessions, we have to consider the presence and protection of primary users in the context of CR networks, which makes the problem more interesting and challenging.

\section{CONCLUdING REMARKS}

Wireless video communication is one of the killer applications for emerging next generation networks. As it is spectrum-hungry and distortion sensitive, intelligent and efficient radio resource management is highly important to meet the demanding performance requirements and also comply with various constraints. This paper investigates the impact of utility function selection on the performance optimization of video communications in CR networks. Two different utility functions have been adopted at a $\mathrm{CE}$ testbed designed for IEEE 802.22 WRAN BS. Our experiment results show that the received video quality at the CPEs can be significantly improved when a video-specific utility function is used as compared to the case when a general purpose utility function is used. The experiment results also show that the improved video quality is achieved at the cost of higher transmit power at the WRAN BS. Although the 802.22 WRAN was used as an example system in this study, we conjecture that the method and general conclusions are applicable to video applications in other types of CR wireless networks as well.

\section{ACKNOWLEDGMENTS}

Youping Zhao's work had been supported in part by Electronics and Telecommunications Research Institute (ETRI), Texas Instruments (TI), and Wireless@ Virginia Tech Partners. He would like to thank Joseph Gaeddert, Lizdabel Morales, and Kyung K. Bae for discussions and collaboration on the development of the WRAN BS CE Testbed at MPRG, Wireless@ Viginia Tech. The work of Jeffrey H. Reed was supported in part by the Wireless@Virginia Tech Partners Program. Shiwen Mao's research is supported in part by the US National Science Foundation under Grant ECCS-0802113 and through the Wireless Internet Center for Advanced Technology (WICAT) at Auburn University. Any opinions, findings, and conclusions or recommendations expressed in this paper are 


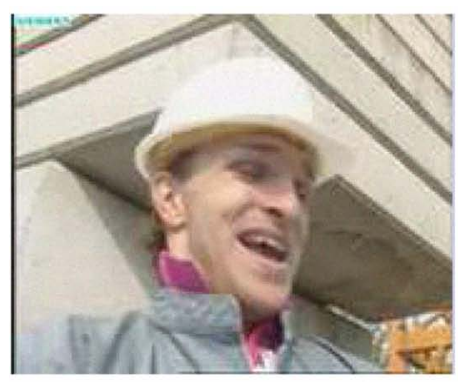

(a) Generic utility function, best case

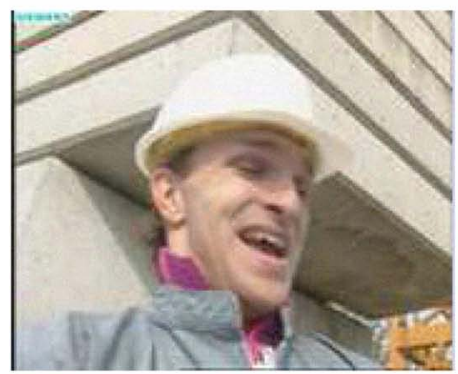

(d) New utility function, best case

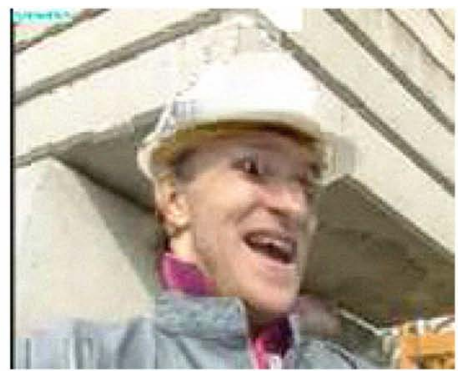

(b) Generic utility function, average case

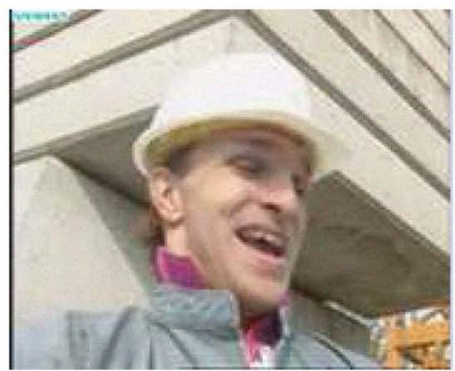

(e) New utility function, average case

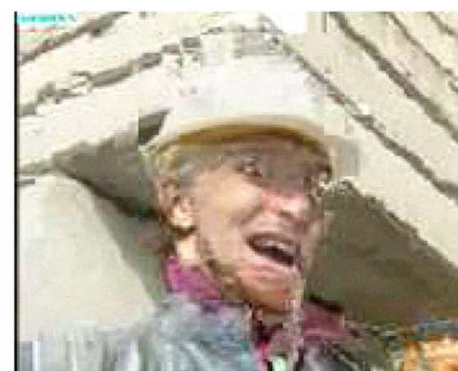

(c) Generic utility function, worst case

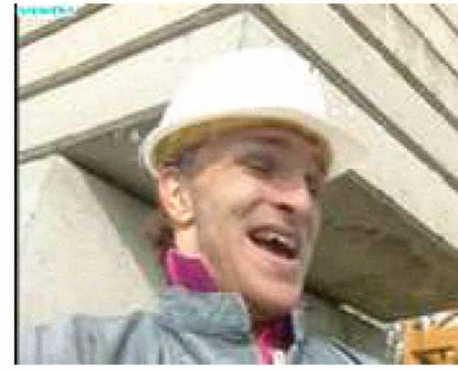

(f) New utility function, worst case

Fig. 11. Visualization of decoded video frames at selected CPEs when different utility functions are used. For both utility functions, the best case occurs at CPE 1, the average case occurs at CPE 7, and the worst case occurs at CPE 3 (see Fig. 7).

those of the authors and do not reflect the position of their sponsors or affiliations.

\section{REFERENCES}

[1] J. Reed, Software Radio-A Modern Approach to Radio Engineering. Upper Saddle River, NJ: Prentice Hall, 2002.

[2] FCC, "Facilitating opportunities for flexible, efficient, and reliable spectrum use employing cognitive radio technologies," Notice of Proposed Rule Making and Order, FCC 03-322, Dec. 2003.

[3] I. Akyildiz, W. Lee, M. Vuran, and S. Mohanty, "Next generation/dynamic spectrum access/cognitive radio wireless networks: A survey," Elsevier Computer Networks, vol. 50, no. 13, pp. 2127-2159, Sept. 2006.

[4] Q. Zhao and B. Sadler, "A survey of dynamic spectrum access - Signal processing, networking, and regulatory policy," IEEE Signal Processing, vol. 24, no. 3, pp. 79-89, May 2007.

[5] IEEE, "Draft standard for wireless regional area networks Part 22 Cognitive wireless RAN medium access control (MAC) and physical layer (PHY) specifications: Policies and procedures for operation in the TV bands," May 2007, IEEE P802.22 Draft Standard (D0.3)

[6] Y. Zhao, S. Mao, J. Neel, and J. H. Reed, "Performance evaluation of cognitive radios: Metrics, utility functions and methodologies," Proceedings of the IEEE, to appear.

[7] J. Gaeddert, L. Morales, Y. Zhao, K. Bae, and J. Reed, "Cognitive engine design for 802.22 WRAN base stations," Blacksburg, VA, June 2007, poster presented at the Wireless@Virginia Tech 2007 Wireless Personal Communications Symposium.

[8] Y. Zhao, J. Gaeddert, L. Morales, K. Bae, and J. Reed, "Development of radio environment map enabled case- and knowledge-based learning algorithms for IEEE 802.22 WRAN cognitive engines," in Proc. the Second International Conference on Cognitive Radio Oriented Wireless Networks and Communications (CROWNCOM 2007), Orlando, FL, Aug. 2007, pp. 44-49.

[9] Y. Zhao, L. Morales, J. Gaeddert, K. Bae, J. Um, and J. Reed, "Applying radio environment map to cognitive wireless regional area networks," in Proc. the Second IEEE International Symposium on Dynamic Spectrum Access Networks (DySPAN 2007), Dublin, Ireland, Apr. 2007, pp. 115118.
[10] K. Stulmuller, N.Farberand, M.Link, and B. Girod, "Analysis of video transmission over lossy channels," IEEE J. Sel. Areas Commun., vol. 18, no. 6, pp. 1012-1032, June 2000.

[11] Y. Wang, Z. Wu, and J. Boyce, "Modeling of transmission-loss-induced distortion in decoded video," IEEE Trans. Circuits Syst. Video Technol., vol. 16, no. 6, pp. 716-732, June 2006.

[12] S. Kompella, S. Mao, Y. Hou, and H. Sherali, "Cross-layer optimized multipath routing for video communications in wireless networks," IEEE J. Sel. Areas Commun., vol. 25, no. 4, pp. 831-840, May 2007.

[13] _ "On path selection and rate allocation for video in wireless mesh networks," IEEE Trans. Networking, to appear.

[14] C. Corderio, K. Challapali, D. Birru, , and S. S. N, "IEEE 802.22: The first worldwide wireless standard based on cognitive radios," in Proc. the 1st IEEE International Symposium on New Frontiers in Dynamic Spectr-um Access Network (DySPAN 2005), Baltimore, MD, Nov. 2005, pp. 328-337.

[15] Y. Zhao, J. Reed, S. Mao, and K. Bae, "Overhead analysis for REMenabled CR networks," in Proc. the First IEEE Workshop on Networking Technologies for Software Defined Radio Networks, Reston, VA, Sept. 2006, pp. 18-25.

[16] Y. Zhao, B. Le, and J. Reed, "Network support - the radio environment map," in Cognitive Radio Technology, B. Fette, Ed. Elsevier, 2006, ch. 11, pp. 337-363.

[17] C. Blum and A. Roli, "Metaheuristics in combinatorial optimization: overview and conceptual comparison," ACM Comput. Surveys, vol. 35 , no. 3, pp. 268-308, Sept. 2003.

[18] T. Chiang and Y. Zhang, "A new rate control scheme using quadratic rate distortion model," IEEE Trans. Circuits Syst. Video Technol., vol. 7, no. 1, pp. 287-311, Feb. 1997.

[19] S. Ma, W. Gao, and Y. Lu, "Rate-distortion analysis for H.264/AVC video coding and its application to rate control," IEEE Trans. Circuits Syst. Video Technol., vol. 15, no. 12, pp. 1533-1544, Dec. 2005.

[20] Z. He and S. Mitra, "A linear source model and a unified rate control algorithm for DCT video coding," IEEE Trans. Circuits Syst. Video Technol., vol. 12, no. 11, pp. 970-982, Nov. 2002.

[21] D. Wu, S. Ci, and H. Wang, "Cross-layer optimization for video summary transmission over wireless networks," IEEE J. Sel. Areas Commun., vol. 25, no. 4, pp. 841-850, May 2007.

[22] G. Wikstrand and J. Sun, "Determining utility functions for streaming 
low bit rate soccer video," Department of Computing Science, Umea University, Tech. Rep., 2004.

[23] C. Sokolowski, M. Petrova, A. de Baynast, and P. Mahonen, "Cognitive radio testbed: Exploiting limited feedback in tomorrow's wireless communication networks," in Proc. IEEE ICC'08 Communications Workshops, Beijing, China, May 2008, pp. 493-498.

[24] J. Park, K. Kim, T. Song, S. Lee, J. Hur, K. Lim, and J. Laskar, "A cross-layer cognitive radio testbed for the evaluation of spectrum sensing receiver and interference analysis," in Proc. IEEE CrownCom'08, Singapore, May 2008, pp. 1-6.

[25] C. Rieser, T. Rondeau, C. Bostian, and T. Gallagher, "Cognitive radio testbed: Further details and testing of a distributed genetic algorithm based cognitive engine for programmable radios," in Proc. IEEE MILCOM'04, Monterey, CA, Oct./Nov. 2004, pp. 1437-1443.

[26] S. Mishra, "A real time cognitive radio testbed for physical and link layer experiments," in Proc. IEEE DySPAN'05, Baltimore, MD, Nov. 2005, pp. 562-567.
[27] M. McHenry, E. Livsics, T. Nguyen, and N. Majumdar, "XG dynamic spectrum access field test results," IEEE Commun., vol. 45, no. 6, pp. 51-57, June 2007.

[28] M. van der Schaar, S. Krishnamachari, S. Choi, and X. Xu, "Adaptive cross-layer protection strategies for robust scalable video transmission over 802.11 WLANs," IEEE J. Sel. Areas Commun., vol. 21, no. 10, pp. 1752-1763, Dec. 2003.

[29] S. Deb, S. Jaiswal, and K. Nagaraj, "Real-time video multicast in WiMAX networks," in Proc. IEEE INFOCOM'08, Phoenix, AZ, Apr. 2008, pp. 1579-1587.

[30] W. Wei and A. Zakhor, "Multiple tree video multicast over wireless ad hoc networks," IEEE Trans. Circuits Syst. Video Technol., vol. 17, no. 1, pp. 2-15, Jan. 2007. 